\title{
Manganese ion chelated FeOCI@PB@PDA@BPQDs nanocomposites as a tumor microenvironment-mediated nanoplatform for enhanced tumor imaging and therapy
}

Zhang, Ming; Sheng, Bulei; Ashley, Jon; Zheng, Tao; Wang, Wentao; Zhang, Qicheng; Zhang, Jun; Zhou, Ninglin; Shen, Jian; Sun, Yi

Published in:

Sensors and Actuators B: Chemical

Link to article, DOI:

10.1016/j.snb.2019.127491

Publication date:

2020

Document Version

Peer reviewed version

Link back to DTU Orbit

Citation (APA):

Zhang, M., Sheng, B., Ashley, J., Zheng, T., Wang, W., Zhang, Q., Zhang, J., Zhou, N., Shen, J., \& Sun, Y. (2020). Manganese ion chelated FeOCl@PB@PDA@BPQDs nanocomposites as a tumor microenvironmentmediated nanoplatform for enhanced tumor imaging and therapy. Sensors and Actuators B: Chemical, 307, [127491]. https://doi.org/10.1016/j.snb.2019.127491

\section{General rights}

Copyright and moral rights for the publications made accessible in the public portal are retained by the authors and/or other copyright owners and it is a condition of accessing publications that users recognise and abide by the legal requirements associated with these rights.

- Users may download and print one copy of any publication from the public portal for the purpose of private study or research.

- You may not further distribute the material or use it for any profit-making activity or commercial gain

- You may freely distribute the URL identifying the publication in the public portal 
Manganese ion chelated FeOCl@PB@PDA@BPQDs Nanocomposites as a Tumor Microenvironment-Mediated Nanoplatform for Enhanced Tumor Imaging and Therapy

Ming Zhang ${ }^{\mathrm{a}, \mathrm{b}^{*}}$, Bulei Sheng ${ }^{\mathrm{c}}$, Jon Ashley ${ }^{\mathrm{a}}$, Tao Zheng ${ }^{\mathrm{a}}$, Wentao Wanga ${ }^{\mathrm{a}^{*}}$, Qicheng Zhang $^{\mathrm{b}}$, Jun Zhang ${ }^{\mathrm{b}}$, Ninglin Zhou ${ }^{\mathrm{b}}$, Jian Shen ${ }^{\mathrm{b}}$, Yi Sun ${ }^{\mathrm{a}^{*}}$

${ }^{a}$ Department of Health Technology, Technical University of Denmark, Kongens Lyngby DK-2800, Denmark;

${ }^{b}$ Jiangsu Collaborative Innovation Center for Biomedical Functional Materials, School of Chemistry and Materials Science, Nanjing Normal University, Nanjing 210023, P. R. China;

${ }^{c}$ Department of Food Science, Aarhus University, 8830 Tjele, Denmark

Corresponding author:Ming Zhang,mzhan@dtu.dk; Yi Sun, suyi@dtu.dk

\begin{abstract}
Herein, a novel tumor microenvironment (TME)-mediated nanotheranostics platform of iron oxychloride $(\mathrm{FeOCl})$ nanorods coated with Prussian Blue (PB), polydopamine (PDA), black phosphorus quantum dots (BPQDs) and chelated with $\mathrm{Mn}^{2+}$ was prepared. In the highly integrated nanoplatform (FeOCl@PB@PDA@BPQDs@Mn), FeOCl catalysts exhibit supreme efficiency to yield hydroxyl radicals $(\bullet \mathrm{OH})$ by $\mathrm{H}_{2} \mathrm{O}_{2}$ decomposition for chemodynamic therapy (CDT). Moreover, the $\mathrm{PB}, \mathrm{FeOCl}$, and $\mathrm{Mn}^{2+}$ have a catalase-like activity that catalyze $\mathrm{H}_{2} \mathrm{O}_{2}$ to release of $\mathrm{O}_{2}$ in the TME. Upon laser irradiation, the BPQDs transform $\mathrm{O}_{2}$ to a singlet oxygen $\left({ }^{1} \mathrm{O}_{2}\right)$ to self-enhance photodynamic therapy (PDT). Additionally, as a result of the high near-infrared (NIR) absorption rate and efficient photothermal conversion of PB and PDA, FeOCl@PB@PDA@BPQDs@Mn nanocomposites (NCs) are capable to work as ideal theranostic agents for photothermal therapy (PTT) in vitro and in vivo. Furthermore, FeOCl@PB@PDA@BPQDs@Mn NCs can also serve as multimodal imaging agents in different methods, such as magnetic resonance (MR), photoacoustic (PA), and ultrasound (US) imaging. Among the tumor models of mice,
\end{abstract}


CDT, PDT, and PTT that combined with multimodal imaging achieved a more significant synergistic therapeutic result compared to any single treatment modality alone. Therefore, the multifunctional nanosystem in this study possesses tremendous potential in providing a satisfying paradigm for effective tumor treatment.

Keywords: tumor microenvironment, chemodynamic therapy, photodynamic therapy, photothermal therapy, multimodal imaging

\section{Introduction}

Black phosphorus quantum dots (BPQDs), which are regarded as metal-free semiconductors, have attracted considerable attention attributed to their unique characteristics, such as a tunable and direct energy band gap, a high photothermal conversion efficient, easy fabrication, excellent biocompatibility, and biodegradation [1-3]. Consequently, plenty of BPQDs have been synthesized from various precursors and applied to many fields, such as sensing [4], photocatalysis [5], photodetectors [6], thin-film solar cells [7], drug carriers [8], and nanomedicine [9]. Particularly in the biomedical areas, it has been proved that small amounts of BPQDs could serve as photosensitizers for PDT [9-11]. BPQDs were able to induce $\mathrm{O}_{2}$ to generate ${ }^{1} \mathrm{O}_{2}$ to kill cancer cells by transferring light energy to $\mathrm{O}_{2}$ [12-14]. However, since the PDT process relies on the release of $\mathrm{O}_{2}$, the rapid consumption of $\mathrm{O}_{2}$ in the microenvironment of ubiquitous hypoxic tumors poses a significant limitation. The rapid proliferation of cancer cells and tumor structural abnormalities in solid tumors would trigger the hypoxic tumor microenvironment (TME) $[15,16]$. As a result, facilitating $\mathrm{O}_{2}$ production in hypoxic solid tumors becomes the crucial aspect in developing BPQDs as an efficient photosensitizer for PDT.

Many attempts have been made in hypoxic cancer cell research to improve PDT efficacy through increasing $\mathrm{O}_{2}$ content among the natural $\mathrm{O}_{2}$ carriers-red blood cells [17]. However, the spontaneous transformation of $\mathrm{O}_{2}$ into $\mathrm{CO}_{2}$ led to a deficiency of $\mathrm{O}_{2}$ [18]. To relieve cancer hypoxia, hyperbaric oxygen therapy could apply as another 
method, but its intrinsic side influence, such as hyperoxic seizures and barotraumas, has limited its usage [15]. Implantable $\mathrm{O}_{2}$-generating depots, such as perfluorocarbon [19], $\mathrm{CaO}_{2}$ [20], and catalase [21], were also fabricated to generate $\mathrm{O}_{2}$ inside the solid tumors [22]. These methods are valuable for PDT by improving hypoxia to some extent, but major drawbacks remain, such as limited biocompatibility, complicated synthesis processes, the short half-life of catalase, as well as the need for exogenous activation [23]. As a result, it becomes essential to design simple and biocompatible nanoplatforms. The novel nanoplatforms should be able to generate $\mathrm{O}_{2}$ continuously without exogenous activation and produce more ${ }^{1} \mathrm{O}_{2}$ under the laser for PDT.

Recently, Mn-based nanostructures and Prussian blue (PB) have been developed to serve as $T_{1}$-contrast agents for magnetic resonance (MR) imaging with high efficiency, as well as catalyze $\mathrm{H}_{2} \mathrm{O}_{2}$ to release $\mathrm{O}_{2}$ within the solid tumor to achieve high PDT or chemodynamic therapy (CDT, the disproportionation of $\mathrm{H}_{2} \mathrm{O}_{2}$ through an intratumoral Fenton reaction) efficacy [18, 24-26]. In the NIR region (650-900 nm), PB could display high absorbance and perfect conversion ability in the charge transfer transition between $\mathrm{Fe}^{2+}$ and $\mathrm{Fe}^{3+}$. These properties facilitated the development of $\mathrm{PB}$ which could serve as a novel near-infrared (NIR) laser-driven photothermal agent for PTT $[27,28]$. As combining PDT and PTT would allow for a very detailed diagnosis and serve as an effective cancer therapy at the same time, we intended to design a new carrier that can integrate BPQDs, $\mathrm{Mn}^{2+}$ and $\mathrm{PB}$ into one nanosystem. Here, we turned our attention to polydopamine (PDA) and novel Fenton-like FeOCl nanorods [29, 30], which had never been applied in cancer therapy. The FeOCl nanorod served as a substrate for $\mathrm{PB}$ coating. Interestingly, we found that $\mathrm{FeOCl}$ nanorods also had the catalase-like activity in catalyzing the decomposition of $\mathrm{H}_{2} \mathrm{O}_{2}$ to yield $\mathrm{O}_{2}$ and $\bullet \mathrm{OH}$. Moreover, the elevated concentration of $\mathrm{O}_{2}$ was helpful to accelerate the generation of ${ }^{1} \mathrm{O}_{2}$ to further enhance the efficacy of PDT.

As a polymer with highly biocompatibility and biodegradability, PDA is synthesized by dopamine self-polymerization in a weakly alkaline environment. Meanwhile, PDA can form a conformal layer by being deposited on nanoparticle (NP) 
surfaces [31, 32]. Moreover, PDA-capped NPs have been utilized to chelate $\mathrm{Mn}^{2+}$ with high efficiency due to its unique features [33, 34]. Besides, attributing to its special functional group and strong NIR absorption, PDA NPs have also been discovered as a platform for loading QDs with high efficiency, as well as photothermal agents for cancer therapy [35]. To make FeOCl@PB@PDA NPs into stable candidates for loading $\mathrm{Mn}^{2+}$ and BPQDs (Scheme 1A), we introduced PDA to modify FeOCl@PB NPs. The obtained FeOCl@PB@PDA@BPQDs@Mn NCs showed satisfying physiological stability, high relaxivity, excellent photothermal performance, and high ${ }^{1} \mathrm{O}_{2} / \cdot \mathrm{OH}$ production. Moreover, the FeOCl@PB@PDA@BPQDs@Mn NCs could generate $\mathrm{O}_{2}$ by catalyzing $\mathrm{H}_{2} \mathrm{O}_{2}$ and ameliorated tumor hypoxia to increase PDT efficiency successfully. By fusing PDT, CDT, PTT, and multi-modal imaging capabilities, in a "one-for-all" nanotheranostics agent, the resultant platform exhibited a remarkably enhanced therapeutic effect (Scheme 1B).
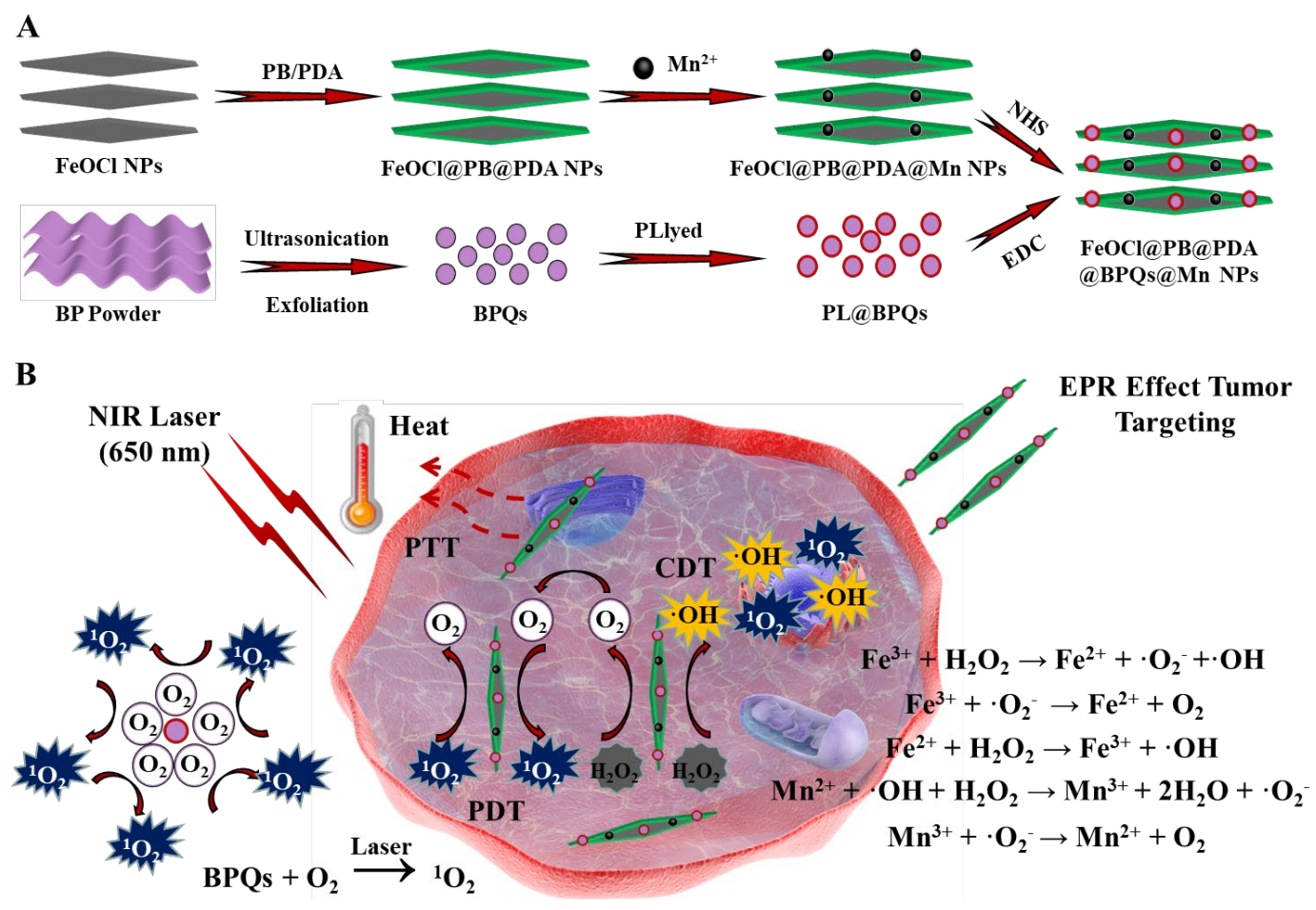

Scheme 1. (A) The preparation route of FeOCl@PB@PDA@BPQDs@Mn nanocomposites. (B) Schematic illustration of FeOCl@PB@PDA@BPQDs@Mn NPs with $\mathrm{O}_{2}$ generation enhancing the PDT, CDT, and PTT under NIR laser irradiation. 


\section{Materials and Methods}

The details of the materials and methods are available in the Supporting Information.

\section{Results and discussion}

\subsection{Preparation and characterization of FeOCI@PB@PDA@BPQDs@Mn NCs}

The process for the design of FeOCl@PB@PDA@BPQDs@Mn NCs was displayed in Scheme 1A. High-resolution transmission electron microscopy (HRTEM), X-ray diffraction (XRD), and X-ray photoelectron spectroscopy (XPS) were applied to characterize the structure of the $\mathrm{FeOCl}$ nanorods. As shown in Figure 1A, TEM images demonstrated that the as-prepared $\mathrm{FeOCl}$ nanorods were typically 40-125 $\mathrm{nm}$ in length and 10-30 $\mathrm{nm}$ in width. FeOCl nanorods displayed high crystalline character, which were proven by the special lattice fringes at 0.343 and $0.309 \mathrm{~nm}$ (Figure 1A) by HRTEM image. These d-spacings were consistent well with $\{011\}$ and $\{110\}$ facets in a formerly confirmed FeOCl crystal structure $[30,36]$. XRD patterns indicated that the chemical composition of the nanorods was $\mathrm{FeOCl}$, which was consistent with the standard card (JCPDS No. 72-0619) [29]. Figure S1 showed XRD peaks appearing at $10.7^{\circ}, 26.1^{\circ}, 35.5^{\circ}$, and $38.2^{\circ}$, which corresponded to the $\{010\},\{110\},\{021\}$, and $\{111\}$ crystal facets, respectively. These results matched well with pure FeOCl crystals. According to XPS (Figure S2), most of the Fe in the $\mathrm{FeOCl}$ nanorods was in the trivalent state. Main peaks at 711.2 and $725.0 \mathrm{eV}$ exhibited a Fe $2 p$ spectrum, which corresponded with the binding energies of $2 p_{3 / 2}$ and $2 \mathrm{p}_{1 / 2}$ in $\mathrm{Fe}^{3+}$, respectively. This matched up with the satellite peaks at 718.6 and $730.1 \mathrm{eV}$ [30]. An additional $\mathrm{Fe} 2 \mathrm{p}_{3 / 2}$ peak discovered at $713.5 \mathrm{eV}$ implied the presence of trace amount of $\mathrm{Fe}^{2+}$ [36]. Nevertheless, Figure S2 shows significant peak for $\mathrm{O} 1 \mathrm{~s}$ and $\mathrm{Cl} 2 \mathrm{p}$.

To prepare the FeOCl@PB core-shell structures, PB nanoshells were deposited onto $\mathrm{FeOCl}$ nanorods under acidic condition. As shown in Figure 1B, the synthesized FeOCl@PB NPs exhibited a well-defined core-shell structure (a PB shell on FeOCl 
nanorods) with thicknesses between 2.5-5 nm. It indicated that $\mathrm{PB}$ had been successfully coated with $\mathrm{FeOCl}$ nanorods. In this study, a PDA shell could form on the surface of FeOCl@PB NPs, because of the self-polymerization of dopamine. As shown in Figure 1C, the core-shell FeOCl@PB@PDA NPs were obtained, with thicknesses between 4-6.5 nm. UV-vis spectra and Fourier transform infrared (FTIR) were applied to verify the successful preparation of FeOCl@PB@PDA NPs. As displayed in Figure S3, the UV-vis absorbance spectrum for the FeOCl@PB@PDA NPs showed a typical absorption peak at around $700 \mathrm{~nm}$, because of the coated PB shell. As shown in Figure S4, the absorption bands of the FTIR spectrum of FeOCl@PB@PDA NPs matched well with FeOCl, PB, and PDA, which confirmed the existence of these components inside the FeOCl@PB@PDA NPs.
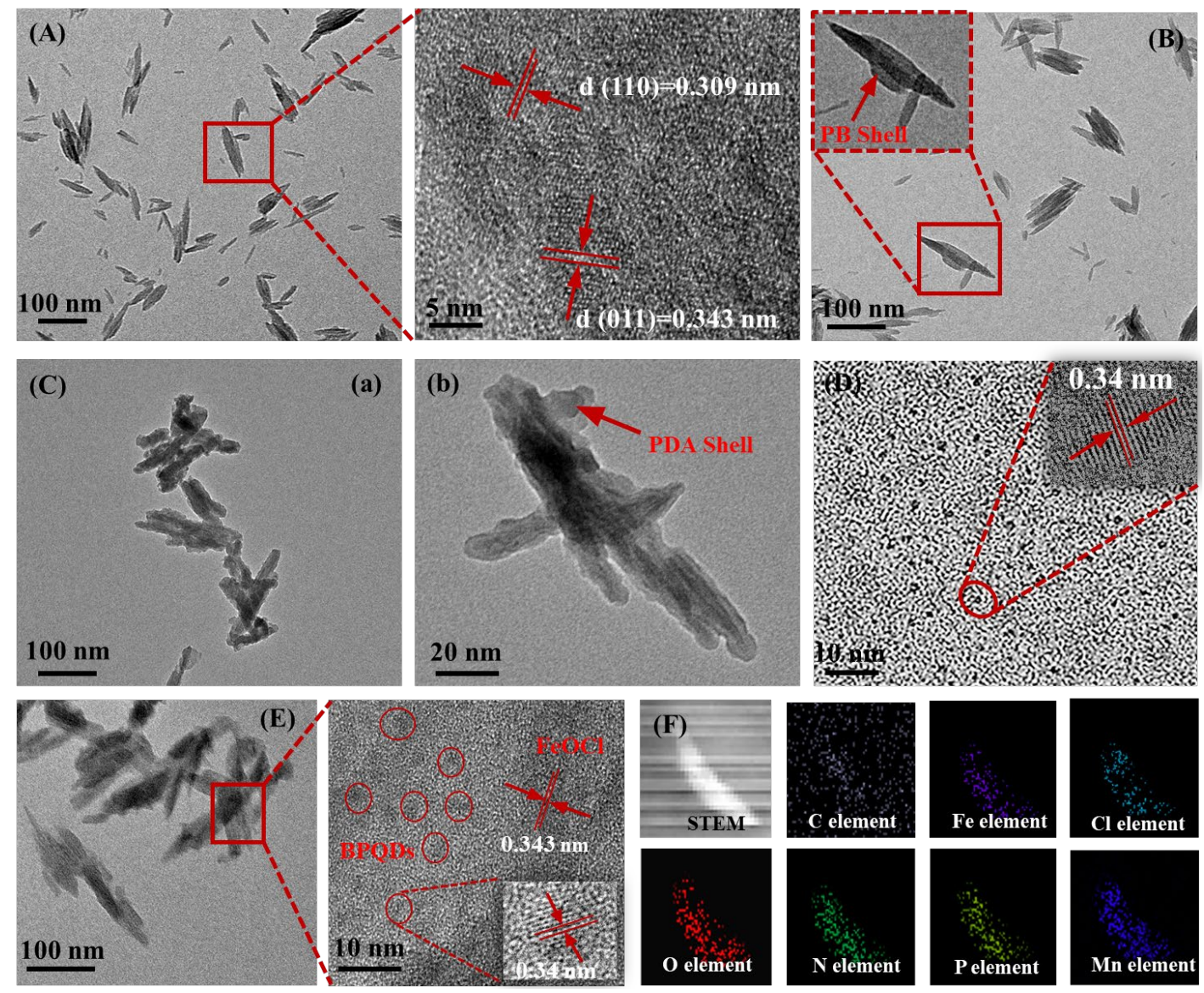

Figure. 1 (A) TEM and HR-TEM images of FeOCl nanorods. (B) TEM images of FeOCl@PB NPs. (C) TEM and HR-TEM images of FeOCl@PB@PDA NPs. (D) TEM images of BPQDs. Inset: HR-TEM images of BPQDs. (E) TEM and HR-TEM images of FeOCl@PB@PDA@BPQDs NPs. Inset: HR-TEM images of BPQDs. (F) HAADF-STEM image and elemental mapping of 
FeOCl@PB@PDA@BPQDs@Mn NCs.

The BPQDs were prepared by liquid ultrasonication exfoliation [2]. The TEM images in Figure 1D clearly showed the uniform nanodots with a size of around $2.8 \pm$ $0.7 \mathrm{~nm}$. The HRTEM image displayed the lattice fringes with an interplane spacing of $0.34 \mathrm{~nm}$, which matched well with the (001) planes of BP crystals. Its crystal structure was also confirmed by XRD (Figure S5). As displayed in Figure S6, the Raman spectrum of BPQDs exhibited three significant peaks at $360.8,437.5$, and $464.9 \mathrm{~cm}^{-1}$. It could be the result of one out-of-plane phonon mode $\left(\mathrm{A}_{\mathrm{g}}{ }^{1}\right)$ and two in-plane modes $\left(\mathrm{B}_{2 \mathrm{~g}}\right.$ and $\left.\mathrm{Ag}^{2}\right)$, respectively. These results also confirmed that the crystalline characteristic of BPQDs still existed after exfoliation from the bulk BP crystal. To obtain BPQDs with carboxyl groups and good biocompatibility, polylysine (PL) was used to modify BPQDs via noncovalent interactions. The result of zeta potential analysis revealed that PL was successfully coated onto the surface of BPQDs (Figure S7). It confirmed that the negative phosphate groups of BPQDs conjugated with the positive amino groups of PL, possibly due to electrostatic interaction.

PL@BPQDs were conjugated to the surface of FeOCl@PB@PDA NPs by performing a EDC/Sulfo-NHS amino coupling reaction to realize its PDT effect. Interestingly, small dense dots were distributed on the surface of FeOCl@PB@PDA NPs uniformly. Figure 1E showed that these small nanodots were BPQDs with an average size of about $2.8 \mathrm{~nm}$. The XPS spectrum showed the existence of elements of P, N, O, Cl, and Fe in the FeOCl@PB@PDA@BPQDs NCs (Figure S8). According to the previous report, the phenolic hydroxyl group could chelate $\mathrm{Mn}^{2+}$ easily [37]. In the system of this study, $\mathrm{Mn}^{2+}$ could be loaded in a mild environment through automatically chelating with the phenolic hydroxyl groups on the PDA. Judging from the corresponding Energy-dispersive X-ray (EDX) figure in Figure 1F, elements of $\mathrm{P}$ and $\mathrm{Mn}$, we could observe a uniform dispersion on the selected area, which indicated that $\mathrm{Mn}^{2+}$ and BPQDs were evenly coated on the surface of FeOCl@PB@PDA NPs. Elemental analysis showed the composition of FeOCl@PB@PDA@BPQDs@Mn as Fe 12.39, Mn 1.14, and P $0.87 \mathrm{wt} \%$. 


\subsection{Photothermal performances of FeOCl@PB@PDA@BPQDs@Mn NCs}

The optical properties of the FeOCl@PB@PDA@BPQDs@Mn NCs solution was studied by the UV-vis-NIR spectroscopy (Figure 2A). The figure exhibited strong and broad NIR absorbance, but no obvious absorption peaks. The property of NIR absorption meant that FeOCl@PB@PDA@BPQDs@Mn NCs could work as a highly promising photothermal agent for PTT. The temperature changes of the solution with differentFeOCl@PB@PDA@BPQDs@Mn NCs concentrations under 1.5 W/cm² of $650 \mathrm{~nm}$ laser irradiation were initially investigated. Upon irradiation, the temperature of the solution increased to $33.2^{\circ} \mathrm{C}(150 \mu \mathrm{g} / \mathrm{mL})$ from $9.3^{\circ} \mathrm{C}(50 \mu \mathrm{g} / \mathrm{mL})$. By comparison, the temperature of the water only control increased by only $2.8^{\circ} \mathrm{C}$ (Figure 2B). The results indicated that the observed temperature increase correlate rapidly with higher concentrations. Previous studies demonstrated that cancer cells can be destroyed in environments of $42^{\circ} \mathrm{C}$ between 15-60 minutes, and the duration time can decrease to 4-6 min when the temperature was greater than $50^{\circ} \mathrm{C}$ [38]. Meanwhile, an IR thermal camera which display temperatures visually was applied to record the real-time thermal imaging of FeOCl@PB@PDA@BPQDs@Mn NCs (inset of Figure 2B). The photothermal conversion efficiency $(\eta)$ of FeOCl@PB@PDA@BPQDs@Mn NCs solution was measured to estimate their ability for photothermal conversion (Figure 2C, D). The $\eta$ value was calculated based on previous reports [38]. The $\eta$ value of FeOCl@PB@PDA@BPQDs@Mn NCs was determined to be $28.4 \%$, which was higher than that of Au nanorods (21\%) [39], Au nanoshells (13\%) [40], $\mathrm{Cu}_{2-\mathrm{x}} \mathrm{Se}(22 \%)$ [41], and $\mathrm{Cu}_{3} \mathrm{BiS}_{3}(27.4 \%)$ [42].

\subsection{H2 $\mathrm{O}_{2}$-responsive FeOCl@PB@PDA@BPQDs@Mn NCs}

$\mathrm{H}_{2} \mathrm{O}_{2}$ and $\cdot \mathrm{OH}$ could oxidise $\mathrm{Mn}^{2+}$ into $\mathrm{Mn}^{3+}$ which efficiently catalyzed the generation of $\mathrm{O}_{2}$ by decomposing $\mathrm{H}_{2} \mathrm{O}_{2}$ molecules [43]. Moreover, $\mathrm{FeOCl}$ nanorods and $\mathrm{PB}$ had catalase-like capability to produce $\mathrm{O}_{2}$ with the decomposition of $\mathrm{H}_{2} \mathrm{O}_{2}$

(Figure 2E and Scheme 1). Therefore, the catalase-like capability of FeOCl@PB@PDA@BPQDs@Mn NCs was tested with $\mathrm{H}_{2} \mathrm{O}_{2}$ solution [43]. It was 
found that FeOCl@PB@PDA@BPQDs@Mn NCs could trigger the $\mathrm{H}_{2} \mathrm{O}_{2}$ solution to generate $\mathrm{O}_{2}$ with a high rate, and obvious bubbles were observed (inset of Figure 2E). Compared to control and $\mathrm{FeOCl}$ nanorods group, the $\mathrm{O}_{2}$ level in $\mathrm{FeOCl} @ \mathrm{~PB}$ and FeOCl@PB@PDA@BPQDs@Mn NCs group increased significantly. The increased $\mathrm{O}_{2}$ level was observed from the decreased fluorescence intensity in the $\mathrm{O}_{2}$ probe $\left(\left[\mathrm{Ru}(\mathrm{dpp})_{3}\right] \mathrm{Cl}_{2}\right) \quad[18], \quad$ suggesting the excellent capability of FeOCl@PB@PDA@BPQDs@Mn NCs to trigger the decomposition of $\mathrm{H}_{2} \mathrm{O}_{2}$. The catalase-like capability to produce $\mathrm{O}_{2}$ of the FeOCl@PB@PDA@BPQDs@Mn NCs was also observed in 4T1 cells (Figure S9).
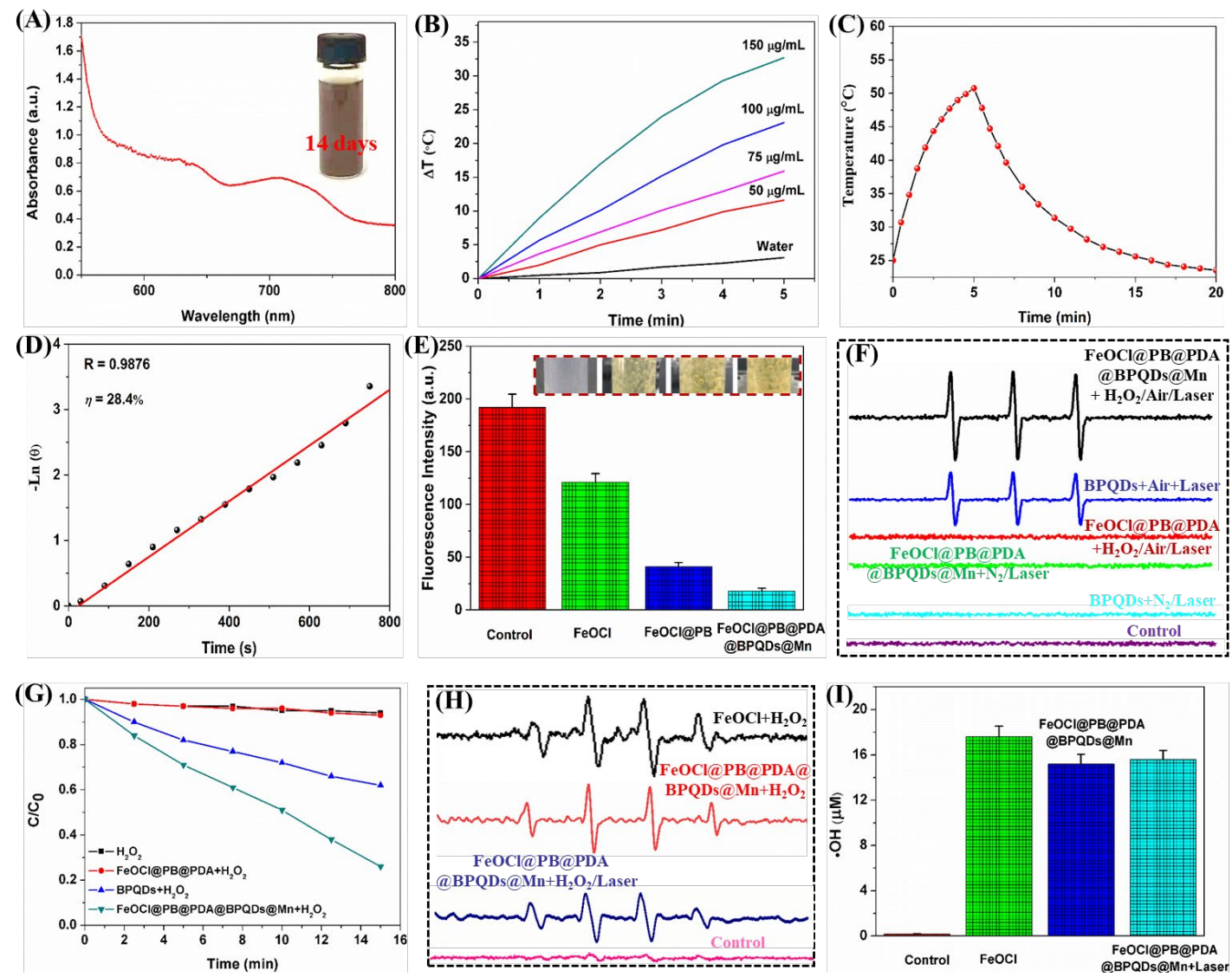

Figure 2. UV-vis absorption spectra of FeOCl@PB@PDA@BPQDs@Mn NCs solution. Inset: The dispersed stability of the FeOCl@PB@PDA@BPQDs@Mn NCs in PBS solution. (B) Temperature elevation of water and FeOCl@PB@PDA@BPQDs@Mn NCs solution with different concentrations under $650 \mathrm{~nm}$ laser $\left(1.5 \mathrm{~W} / \mathrm{cm}^{2}\right)$ irradiation for $5 \mathrm{~min}$. Inset: IR graphs of FeOCl@PB@PDA@BPQDs@Mn NCs with different concentrations. (C) The photothermal response of FeOCl@PB@PDA@BPQDs@Mn NCs solution (100 $\mu \mathrm{g} / \mathrm{mL})$ under 650 nm laser irradiation and then the laser was shut off. (D) The linear relationship between time and $-\operatorname{Ln} \Theta$ obtained from the cooling time of $(\mathrm{C})$. (E) The generation of average fluorescence intensity of $\left[\mathrm{Ru}(\mathrm{dpp})_{3}\right] \mathrm{Cl}_{2}$-added 
mixture containing $\mathrm{H}_{2} \mathrm{O}_{2}$ and PBS, FeOCl, FeOCl@PB or FeOCl@PB@PDA@BPQDs@Mn NCs, respectively. (F) EPR spectra of different reaction systems with the TEMP as the spin trap under 650 $\mathrm{nm}$ laser irradiation. $(\mathrm{G})$ Normalized absorbance of the DPBF in the presence of different reaction systems. (H) EPR spectra of different reaction systems with the BMPO as the spin trap. (I) Cumulative $\cdot \mathrm{OH}$ formation in the presence of different reaction systems.

\subsection{The detection of ROS generation}

As a result of the effective production of $\mathrm{O}_{2}$ coming from $\mathrm{H}_{2} \mathrm{O}_{2}$ triggered by the FeOCl@PB@PDA@BPQDs@Mn NCs, the electron spin resonance (ESR) technique with 2,2,6,6-tetramethylpiperidine (TEMP) as the ${ }^{1} \mathrm{O}_{2}$ trapper was applied to detect the 1을 -generation capability of the FeOC1@PB@PDA@BPQDs@Mn NCs with the existence of acidic $\mathrm{H}_{2} \mathrm{O}_{2}$ (Figure 2F). In the atmosphere of $\mathrm{N}_{2}$, the FeOCl@PB@PDA@BPQDs@Mn NCs and BPQDs solution exhibited no specifical ${ }^{1} \mathrm{O}_{2}$-induced signal with the irradiation of $650 \mathrm{~nm}$ laser $\left(0.1 \mathrm{~W} / \mathrm{cm}^{2}, 5 \mathrm{~min}\right)$, because the supply of $\mathrm{O}_{2}$ was not sufficient. By contrast, a strong signal was detected in the FeOCl@PB@PDA@BPQDs@Mn NCs solution with the presence of acidic $\mathrm{H}_{2} \mathrm{O}_{2}$ buffer. However, the BPQDs showed a relatively weak characteristic ${ }^{1} \mathrm{O}_{2}$-induced signal. It has been reported that the photogenerating capability of ${ }^{1} \mathrm{O}_{2}$ can be measured by probe molecules during particular reactions, such as the 1,3-diphenylisobenzofuran (DPBF) which can react with ${ }^{1} \mathrm{O}_{2}$ through Diels-Alder 1,4-cycloaddition to show a reduced absorption intensity at around $410 \mathrm{~nm}$. Therefore, the time-dependent photodegradation of DPBF exposed to the $650 \mathrm{~nm}$ laser $\left(0.1 \mathrm{~W} / \mathrm{cm}^{2}\right)$ was monitored to evaluate the ${ }^{1} \mathrm{O}_{2}$ generation capability of FeOCl@PB@PDA@BPQDs@Mn NCs. Figure S10 displays that the absorption of DPBF at $410 \mathrm{~nm}$ decreased significantly in the presence of FeOCl@PB@PDA@BPQDs@Mn NCs, which suggested the high production of ${ }^{1} \mathrm{O}_{2}$ with prolonged time during irradiation. Figure $2 \mathrm{G}$ showed that pure DPBF did not decompose upon irradiation with NIR light at $650 \mathrm{~nm}$. The slight increase in ROS generation capability of FeOCl@PB@PDA@BPQDs@Mn NCs when compared to other BPQDs might be due to the catalase-like activity of $\mathrm{FeOCl}$ nanorods, $\mathrm{PB}$, and $\mathrm{Mn}^{2+}$.

In this study, 5-tertbutoxycarbonyl-5-methyl-1-pyrroline N-oxide (BMPO) was 
selected to serve as a spin trap for the $\bullet \mathrm{OH}$. As shown in Figure $2 \mathrm{H}$, an $\bullet \mathrm{OH}$-induced signal of the same intensity was observed in the $\mathrm{FeOCl}$ nanorods, FeOCl@PB@PDA@BPQDs@Mn NCs, and FeOCl@PB@PDA@BPQDs@Mn NCs, when they were exposed to the $650 \mathrm{~nm}$ laser $\left(0.1 \mathrm{~W} / \mathrm{cm}^{2}\right)$. Therefore, to investigate the capability of FeOCl@PB@PDA@BPQDs@Mn NCs for·OH generation, the total amount of $• \mathrm{OH}$ in the mixed solution of FeOCl@PB@PDA@BPQDs@Mn NCs/acidic $\mathrm{H}_{2} \mathrm{O}_{2}$ was measured. The total amount of $\bullet \mathrm{OH}$ was similar in the presence of FeOCl nanorods, FeOCl@PB@PDA NPs, and FeOCl@PB@PDA@BPQDs@Mn NCs. These results confirmed that $\bullet \mathrm{OH}$ was generated by $\mathrm{FeOCl}$ nanorods, which was consistent with a previous report [29].

Intracellular ROS production was evaluated with a cell-permeable fluorescent dye dichlorofluorescein (DCF) [3,11]. Figure 3A shows that the incubation of BPQDs without $650 \mathrm{~nm}$ laser irradiation exhibited limited fluorescence of DCF, but stronger green fluorescence in cells was detected after irradiation under $650 \mathrm{~nm}$ laser. This result indicated that the production of ROS inside the cells during the irradiation. It exhibited similar fluorescence of DCF when $\mathrm{FeOCl}$ nanorods solution (without or with $650 \mathrm{~nm}$ laser irradiation) was incubated after acidic $\mathrm{H}_{2} \mathrm{O}_{2}$ buffer was added, which revealed that the influence of laser irradiation on the generation of ROS inside the cells was limited. Cells dealt with FeOCl@PB@PDA@BPQDs@Mn NCs exhibited weak green fluorescence, while the cells treated with FeOCl@PB@PDA@BPQDs@Mn NCs showed stronger green fluorescence after irradiation with the laser. It illustrated that FeOCl@PB@PDA@BPQDs@Mn NCs worked as the catalyst for $\bullet \mathrm{OH}$ generation from $\mathrm{H}_{2} \mathrm{O}_{2}$ activation. As expected, cells incubated byFeOCl@PB@PDA@BPQDs@Mn NCs (after650 nm laser irradiation) with the addition of an acidic $\mathrm{H}_{2} \mathrm{O}_{2}$ exhibited inordinately bright green fluorescence. The dramatically higher ROS production ability of FeOCl@PB@PDA@BPQDs@Mn $\mathrm{NCs}$ than for $\mathrm{FeOCl}$ nanorods or BPQDs which may be the result of the dual-modal ROS generation by $\mathrm{FeOCl}$ nanorods and BPQDs.

\subsection{Biocompatibility of FeOCl@PB@PDA@BPQDs@Mn NCs}


Hemolysis means the destruction of membrane integrity of red blood cells (RBCs), as well as the consequently release of hemoglobin from RBCs [44]. The extent of hemolysis in RBCs under different concentrations of FeOCl@PB@PDA@BPQDs@Mn NCs in this work was also investigated (Figure S11). The obtained results showed that the largest hemolytic percentage of FeOCl@PB@PDA@BPQDs@Mn NCs was about 0.082\%. Meanwhile, the visual inspection of specific digital photographs was applied to confirm the low hemolytic efficiency of FeOCl@PB@PDA@BPQDs@Mn NCs. This result suggested that the effect of the FeOCl@PB@PDA@BPQDs@Mn NCs on hemolysis was limited. The interaction and incompatibility of samples with RBCs can refer to aggregation, crenation, and hemolysis. From Figure 3B, it could be concluded that the RBCs still exhibited natural morphology with normal biconcave shapes, which was the same as that in PBS, even when the cells were in the presence of high concentrations (500 $\mu \mathrm{g} / \mathrm{mL})$ of FeOCl@PB@PDA@BPQDs@Mn NCs. These results matched well with the analysis of hemolysis above.
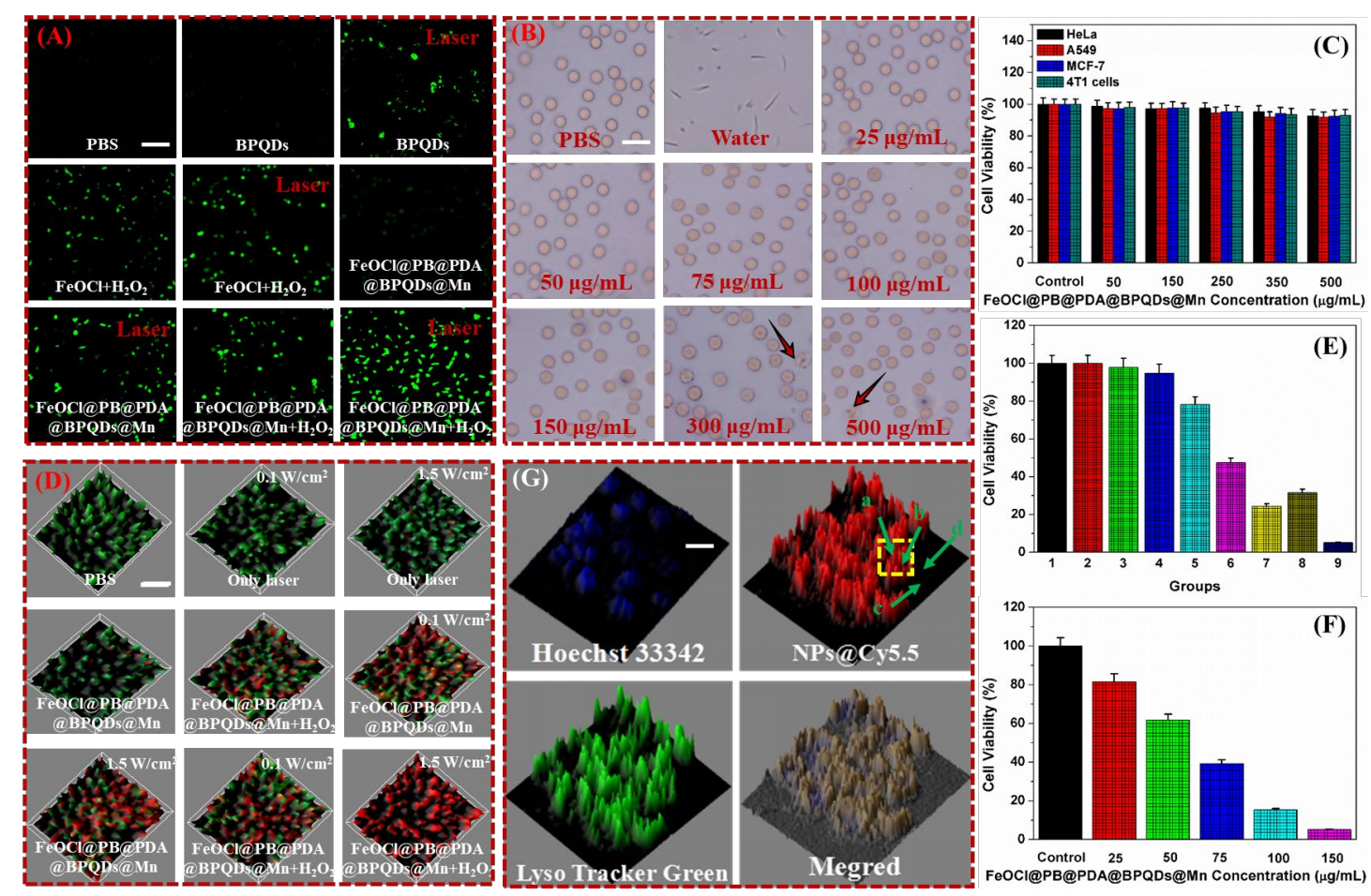

Figure 3. (A) Fluorescence images of DCF stained $4 \mathrm{~T} 1$ cells in the presence of different reaction systems. Size bar $=100 \mu \mathrm{m}$. (B) Morphology images of $\mathrm{RBCs}$ incubated with 
FeOCl@PB@PDA@BPQDs@Mn NCs at concentrations of 0-500 $\mu \mathrm{g} / \mathrm{mL}$. Size bar = $10 \mu \mathrm{m}$. (C) Cell viability of HeLa, 4T1, A549, and MCF7 cells after incubation with FeOCl@PB@PDA@BPQDs@Mn NCs at concentrations of 0-150 $\mu \mathrm{g} / \mathrm{mL}$ for $24 \mathrm{~h}$. (D) Fluorescence images of calcein AM/PI-stained 4T1 cells incubated with different reaction systems. Size bar $=100 \mu \mathrm{m}$. (E) Relative viability of different groups in (D). (F) Cell viability of 4T1 cells after incubation with FeOCl@PB@PDA@BPQDs@Mn NCs at concentrations of $0-150 \mu \mathrm{g} / \mathrm{mL}$ under $650 \mathrm{~nm}$ laser $\left(1.5 \mathrm{~W} / \mathrm{cm}^{2}\right)$ irradiation for $5 \mathrm{~min}$. (G) Colocalization analysis of Cy5.5-labelled FeOCl@PB@PDA@BPQDs@Mn NCs in 4T1 cells. Size bar $=20 \mu \mathrm{m}$.

The ideal therapeutic agent should have little to no toxicity in the human body. Therefore, we examined the cytotoxicity of the FeOCl@PB@PDA@BPQDs@Mn NCs in various cell lines. The standard assay of methyl thiazolyl tetrazolium (MTT) was applied to determine the cell viability rate of different cell lines after incubation under different concentrations of FeOCl@PB@PDA@BPQDs@Mn NCs for 24 h (Figure 3C). In short, no obvious cytotoxicity was detected in any of the examined cell lines, even in the presence of high concentrations up to $150 \mu \mathrm{g} / \mathrm{mL}$. This discovery indicated that FeOCl@PB@PDA@BPQDs@Mn NCs had great potential in biomedical applications with satisfying biocompatibility and suitability.

\subsection{In vivo anticancer treatment}

The prepared FeOCl@PB@PDA@BPQDs@Mn NCs featured efficient NIR laser irradiation to induce the generation of ${ }^{1} \mathrm{O}_{2}, \cdot \mathrm{OH}$, and heat. For imaging-guided CD, PDT, and PTT, these properties might promote the application of FeOCl@PB@PDA@BPQDs@Mn NCs to serve as a multimodal and NIR laser triggered cancer theranostic agent. Then, the efficacy of bimodal PDT/PTT and cytotoxicity of the prepared FeOCl@PB@PDA@BPQDs@Mn NCs in vitro were studied. The method of calcein AM (green) and PI (red) co-staining can be applied to identify live and dead cells $[11,44]$. In PBS buffer, laser $\left(0.1\right.$ or $\left.1.5 \mathrm{~W} / \mathrm{cm}^{2}\right)$ only, and FeOCl@PB@PDA@BPQDs@Mn NCs groups, no dead cells were found, because all observed cells retained their green fluorescence (Figure 3D). This result confirmed the good biocompatibility of FeOCl@PB@PDA@BPQDs@Mn NCs and illustrated that pure water under irradiation could not generate heat to destroy cancer cells. However, with the addition of acidic $\mathrm{H}_{2} \mathrm{O}_{2}$ buffer or laser irradiation of $0.1 \mathrm{~W} / \mathrm{cm}^{2}$, some cells 
were killed after being incubated with 150 rg/mLFeOCl@PB@PDA@BPQDs@Mn NCs because of the release of $\bullet \mathrm{OH}$ or ${ }^{1} \mathrm{O}_{2}$, even though, no heat was generated under laser irradiation at low power doses [45]. With $1.5 \mathrm{~W} / \mathrm{cm}^{2}$ of laser irradiation or the addition of acidic $\mathrm{H}_{2} \mathrm{O}_{2}$ buffer/ $0.1 \mathrm{~W} / \mathrm{cm}^{2}$ of laser irradiation, a significant number of cells were destroyed, as displayed by the stronger red fluorescence of cells. By contrast, all cells were killed essentially with laser irradiation of $2.0 \mathrm{~W} / \mathrm{cm}^{2}$ or the addition of acidic $\mathrm{H}_{2} \mathrm{O}_{2}$ buffer, as shown by the intensive and homogeneous red fluorescence of cells. This discovery was the result of the fact that when a $650 \mathrm{~nm}$ laser was applied, FeOCl@PB@PDA@BPQDs@Mn NCs could simultaneously

generate $\cdot \mathrm{OH},{ }^{1} \mathrm{O}_{2}$, and produce heat at high laser power doses to kill cells by CDT, PDT, and PTT efficiently at the same time. We further evaluated the therapeutic efficacy of FeOCl@PB@PDA@BPQDs@Mn NCs quantitatively by MTT assay. In Figure 3E, the inhibitory effect of the MTT assay on cells matched well with the result of the dye experiment in vitro. It could be emphasized that therapeutic efficacy depends on the concentration of FeOCl@PB@PDA@BPQDs@Mn NCs.Cytotoxicity in cells became obvious when the concentration of FeOCl@PB@PDA@BPQDs@Mn $\mathrm{NCs}$ was more than $50 \mu \mathrm{g} / \mathrm{mL}$ (Figure 3F). These results revealed that FeOCl@PB@PDA@BPQDs@Mn NCs were highly biocompatible and could be applied for cancer-specific and self-enhanced CDT, PDT, and PTT.

\subsection{In vitro cellular uptake}

To confirm the intracellular locations and relative amounts of Cy5.5-labelled FeOCl@PB@PDA@BPQDs@Mn NCs, co-staining tests with lysosomal tracker and nucleus marker were performed [46]. As shown in Figure 3G, the fluorescence images of 4T1 cells incubated with Cy5.5-labelled FeOCl@PB@PDA@BPQDs@Mn NCs showed obvious bright red fluorescence, which efficiently overlaid with the green fluorescence coming from the lysosomal tracker. This implied that Cy5.5-labelled FeOCl@PB@PDA@BPQDs@Mn NCs mainly accumulated in the lysosomes of 4T1 cells. Meanwhile, fluorescence images and the overlay of the bright field images implied that the luminescence was from the inside of 4T1 cells. To present the 
fluorescence signals for the living cells labeled with Cy5.5-labelled FeOCl@PB@PDA@BPQDs@Mn NCs, a quantitative analysis of the intensity of the fluorescence and the background was performed. Four specific spots were selected to analyze the intensity of fluorescence signals by ImageJ. The counts of the fluorescence signal intensities of spot $\mathrm{a}$ and $\mathrm{b}$ exceeded or were close to the detection of about 97, whereas the counts of the background noise at spot $\mathrm{c}$ and d were about 6 .

\subsection{In vivo imaging and biodistribution}

Before the in vivo combination therapy, the characteristics of FeOCl@PB@PDA@BPQDs@Mn NCs in vivo were confirmed. Female Balb/c mice were injected with FeOCl@PB@PDA@BPQDs@Mn NCs intravenously. Their blood was collected at each time point. After being diluted in lysis buffer and extracted by $\mathrm{HCl} /$ isopropanol, Inductively Coupled Plasma-Atomic Emission Spectrometry (ICP-AES) was performed to detect the concentration of $\mathrm{Mn}^{2+}$ in the blood. Figure $\mathrm{S} 12$ indicates that the signal of $\mathrm{Mn}^{2+}$ gradually decreased over time, which followed a two-compartment model. The first $\left(\mathrm{t}_{1 / 2(\alpha)}\right)$ and second $\left(\mathrm{t}_{1 / 2(\beta)}\right)$ phases of circulation half-lives were confirmed to be $2.9 \pm 0.14 \mathrm{~h}$ and $9.4 \pm 0.92 \mathrm{~h}$, respectively.

Previous studies have reported that $\mathrm{Mn}^{2+}$ and $\mathrm{PB}$ could support a clear contrast under T1-weighted MR imaging [27, 28]. In this study, FeOCl@PB@PDA@BPQDs@Mn NCs showed an enhanced lightening in T1-weighted MR imaging. Figure 4A, B displayed the in vitro T1 relaxation time of both FeOCl@PB@PDA@BPQDs and FeOCl@PB@PDA@BPQDs@Mn NCs solution after shaking for $24 \mathrm{~h}$, which indicated that the relaxation time of different concentrations of FeOCl@PB@PDA@BPQDs@Mn NCs was much shorter than that of FeOCl@PB@PDA@BPQDs NCs. This result implied better T1-weighted MR imaging performance of FeOCl@PB@PDA@BPQDs@Mn NCs than that of FeOCl@PB@PDA@BPQDs NCs. The concentration-dependent longitudinal relaxivity (r1) value of FeOCl@PB@PDA@BPQDs@Mn NCs was confirmed to be $15.57 \mathrm{mM}^{-1} \mathrm{~S}^{1}$ (Figure 4A), which was higher than that of the previous report (14.15 $\mathrm{mM}^{-1} \mathrm{~S}^{1}$ ) for the Mn-chelated PDA NPs [33]. In vivo experiments were applied to 
evaluate the T1 weighted MR imaging capability of both FeOCl@PB@PDA@BPQDs and FeOCl@PB@PDA@BPQDs@Mn NCs. It was found that T1-weighted MR imaging performance of FeOCl@PB@PDA@BPQDs NCs showed only a slightly higher contrast improvement for tumors in the time course of post-injection (Figure 4C, a). However, the remarkable enhancement of T1-weighted MR imaging contrast of the tumor could be observed after the injection ofFeOCl@PB@PDA@BPQDs@Mn NCs (Figure 4C, b). In detail, the T1-weighted MR imaging signal intensity of tumor site increased to $527 \pm 19.1$ after injection with FeOCl@PB@PDA@BPQDs@Mn NCs (Figure S13), which confirmed that FeOCl@PB@PDA@BPQDs@Mn NCs was an excellent Mn-based T1-weighted MR imaging contrast agents in vivo. Afterward, ICP-AES measurements of $\mathrm{Mn}^{2+}$ levels in different organs was performed to further determine the biodistribution of FeOCl@PB@PDA@BPQDs@Mn NCs. At 24 h post-injection, the tumor uptake of NCs was confirmed to be relatively high at about $11.2 \% \mathrm{ID} / \mathrm{g}$. This passive tumor accumulation of NCs could be a result of the improved permeability and retention (EPR) effect of tumor tissues (Figure S14).
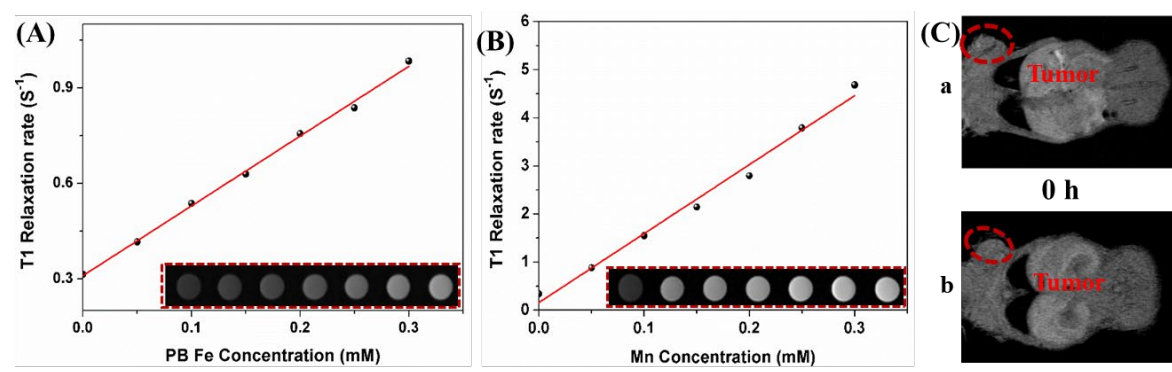

$\mathbf{0 ~ h}$
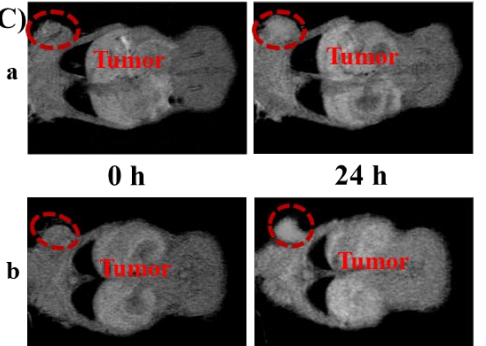

$24 \mathrm{~h}$

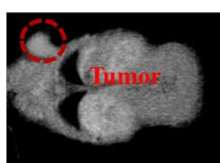

(F)
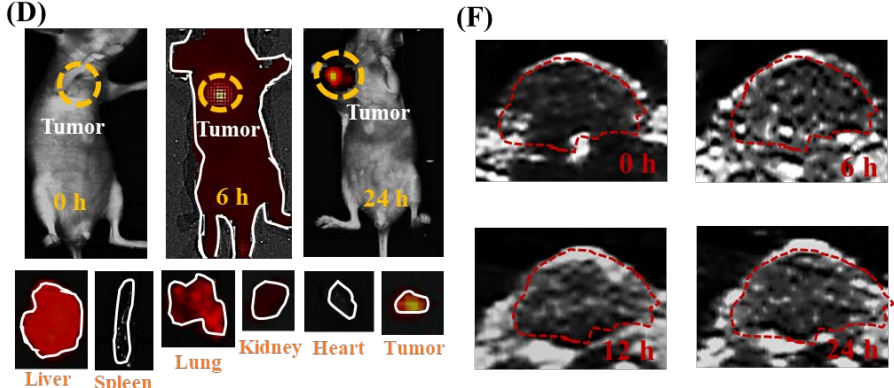

(G) 0 min $\quad 1$ min $\quad 2$ min $\quad 3$ min $\quad 4$ min $\quad 5$ min
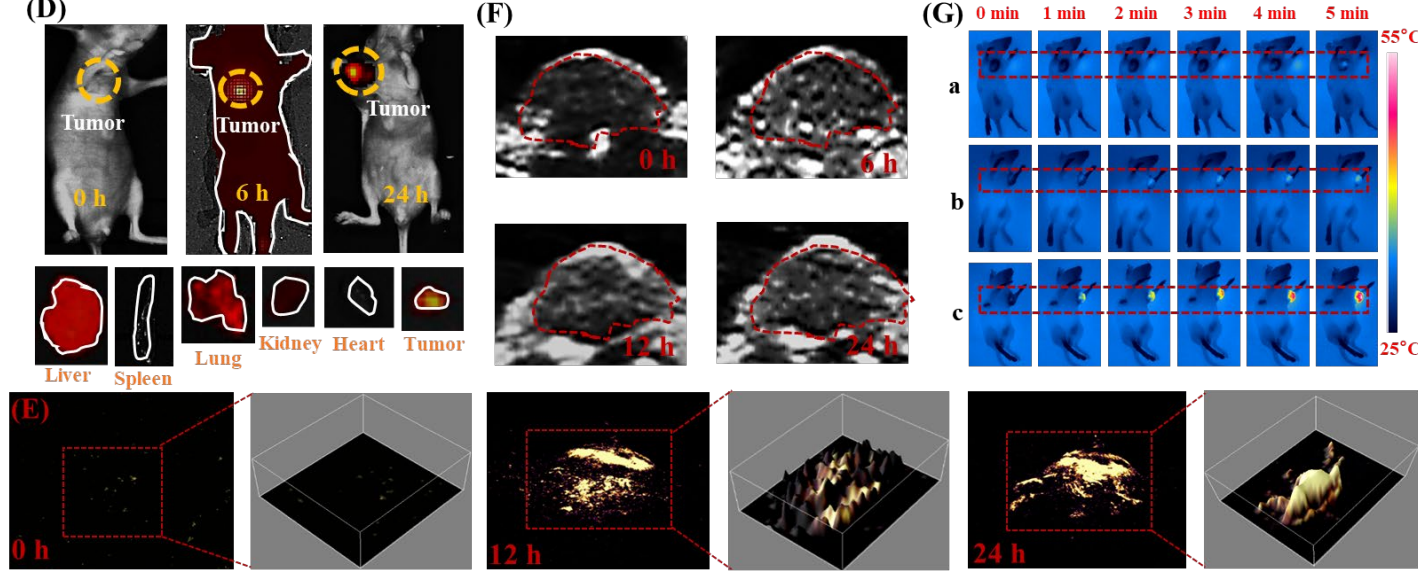

Figure 4. (A) T1 relaxation rates of FeOCl@PB@PDA@BPQDs NCs dispersions at different Fe iron 
concentrations. Inset: T1-MR images of FeOCl@PB@PDA@BPQDs NCs with different Fe iron concentrations. (B) T1 relaxation rates of FeOCl@PB@PDA@BPQDs@Mn NCs dispersions at different $\mathrm{Mn}^{2+}$ concentrations. Inset: T1-MR images of FeOCl@PB@PDA@BPQDs@Mn NCs with different $\mathrm{Mn}^{2+}$ concentrations. (C) T1-weighted MR images of 4T1 tumor-bearing mice treated with (a) FeOCl@PB@PDA@BPQDs and (b) FeOCl@PB@PDA@BPQDs@Mn NCs. (D) Fluorescence images of 4T1 tumor-bearing mice treated with Cy5.5-labelled FeOCl@PB@PDA@BPQDs NCs. (E) PA imaging of 4T1 tumor-bearing mice treated with FeOCl@PB@PDA@BPQDs NCs. (F) US imaging monitoring the generation of $\mathrm{O}_{2}$ in tumors. (G) The IR thermal camera images of 4T1 tumor-bearing mice treated with (a) PBS exposed with NIR laser $\left(1.5 \mathrm{~W} / \mathrm{cm}^{2}, 5 \mathrm{~min}\right)$, (b) FeOCl@PB@PDA@BPQDs NCs exposed with 0.1 W/ $/ \mathrm{cm}^{2}$ NIR laser, and (c) 1.5 W/ $/ \mathrm{cm}^{2}$ NIR laser.

Fluorescence imaging was applied directly to assess the biodistribution of the Cy5.5-labelledFeOCl@PB@PDA@BPQDs@Mn NCs in the mice. Figure 4D shows that extensive fluorescence could be detected from the tumor at $6 \mathrm{~h}$ post-injection and the subcutaneous tumor could be easily determined from other tissues. The fluorescence intensity in the tumor at $24 \mathrm{~h}$ indicated that the FeOCl@PB@PDA@BPQDs@Mn NCs could accumulate at the tumor site. Afterward, these mice were sacrificed at $24 \mathrm{~h}$ post-injection, and their major organs were collected for fluorescence imaging ex vivo (Figure 4D). The clear fluorescence could be detected from the tumor and some organs including the kidney, liver, and lung (Figure 4D and Figure S15).

As the latest designed and combined biomedical imaging modality, PA imaging detects the ultrasonic waves coming from tissues absorption of a pulsed laser. As shown in Figure S16,FeOCl@PB@PDA@BPQDs@Mn NCs showed obvious PA contrast effect, which encouraged us to perform PA imaging to verify the high accumulation of NCs in the tumor site. As shown in Figure 4E, no PA signal could be observed withoutFeOCl@PB@PDA@BPQDs@Mn NCs. After the injection for 12 h, the intensity of PA signal in the tumor site increased and the morphology of the tumor became more obvious, indicating the accumulation of FeOCl@PB@PDA@BPQDs@Mn NCs at the tumor site. The PA signals were enhanced after24 h injection withFeOCl@PB@PDA@BPQDs@Mn NCs. It should be noted that the accumulation behavior of FeOCl@PB@PDA@BPQDs@Mn NCs confirmed by the in vivo PA imaging matched well with the results of MR and 
fluorescence imaging.

The production capability of $\mathrm{O}_{2}$ in the tumor was evaluated by US imaging $[47,48]$. Figure $4 \mathrm{~F}$ indicates an increasing level of $\mathrm{O}_{2}$, observed over time in the tumor site after the mice were injected with FeOCl@PB@PDA@BPQDs@Mn NCs. The production of $\mathrm{O}_{2}$ in the tumor was quantified by the echo intensity and most $\mathrm{O}_{2}$ was determined at $24 \mathrm{~h}$. Therefore, the Fenton reaction with $\mathrm{H}_{2} \mathrm{O}_{2}$ is time-dependent with a slow rate. Compared with the $\mathrm{O}_{2}$ amount before injection, an increase of 2.25 fold in $\mathrm{O}_{2}$ levels in the FeOCl@PB@PDA@BPQDs@Mn NCs-treated tumor was observed (Figure S17). Afterward, it was further revealed that the time point of $24 \mathrm{~h}$ was appropriate for PDT because of the optimum levels of $\mathrm{O}_{2}$ that were produced by FeOCl@PB@PDA@BPQDs@Mn NCs at the tumor site over that time point. This result enhanced the therapeutic effect of PDT.

\subsection{In vivo CDT, PDT, and PTT treatments}

In the light of photothermal conversion of FeOCl@PB@PDA@BPQDs@Mn NCs, in vivo PTT treatment with FeOCl@PB@PDA@BPQDs@Mn NCs in the 4T1-bearing tumor mice model were carried out. Figure 4G and Figure S18 indicated that upon irradiation at the power densities of $1.5 \mathrm{~W} / \mathrm{cm}^{2}$, the temperature of tumor site increased to $51.3^{\circ} \mathrm{C}$ within $5 \mathrm{~min}$, which was high enough to destroy cancer cells in vivo. As control groups (PBS+1.5 W/cm² and FeOCl@PB@PDA@BPQDs@Mn $\mathrm{NCs}+0.1 \mathrm{~W} / \mathrm{cm}^{2}$ ), the tumor temperature was slightly altered. These results indicated that FeOCl@PB@PDA@BPQDs@Mn NCs could be applied as a PTT agent for in vivo tumor destruction with high efficiency. Then, the in vivo CDT, PDT, and PTT therapeutic efficacy of FeOCl@PB@PDA@BPQDs@Mn NCs were studied. The 4T1 tumor-bearing mice were divided into four groups randomly. As a treated group, the mice were intravenously injected with FeOCl@PB@PDA@BPQDs@Mn NCs and followed the irradiation under $650 \mathrm{~nm}$ laser of $1.5 \mathrm{~W} / \mathrm{cm}^{2}$ for $5 \mathrm{~min}$. For the groups, the mice were administered with PBS or FeOCl@PB@PDA@BPQDs@Mn NCs without or with $0.1 \mathrm{~W} / \mathrm{cm}^{2}$ laser irradiation. The tumor sizes were measured by a 
digital caliper every two days. Figures 5 A,B and Figure S19 indicate that the tumors were ablated effectively and only black scars remained at tumor sites, after the injection of FeOCl@PB@PDA@BPQDs@Mn NCs and laser irradiation of 1.5 $\mathrm{W} / \mathrm{cm}^{2}$. Tumor ablation could be observed after the mice were administered with FeOCl@PB@PDA@BPQDs@Mn NCs both with or without 0.1 W/cm² laser irradiation, and the tumor showed only a short delay during tumor growth. These results suggested that the single PDT or single CDT/PDT effect of FeOCl@PB@PDA@BPQDs@Mn NCs could not kill tumors completely. In contrast, after the PBS injection, tumors grew quickly within the test period. Additionally, staining images of tumor sites treated by H\&E and TUNLE are shown in Figures 5C. The morphology with distinctive membrane and nuclear structures of the cancer cells in control groups did not change significantly, while the cancer cells were destroyed obviously in the group treated with FeOCl@PB@PDA@BPQDs@Mn NCs injection under laser irradiation of $1.5 \mathrm{~W} / \mathrm{cm}^{2}$.
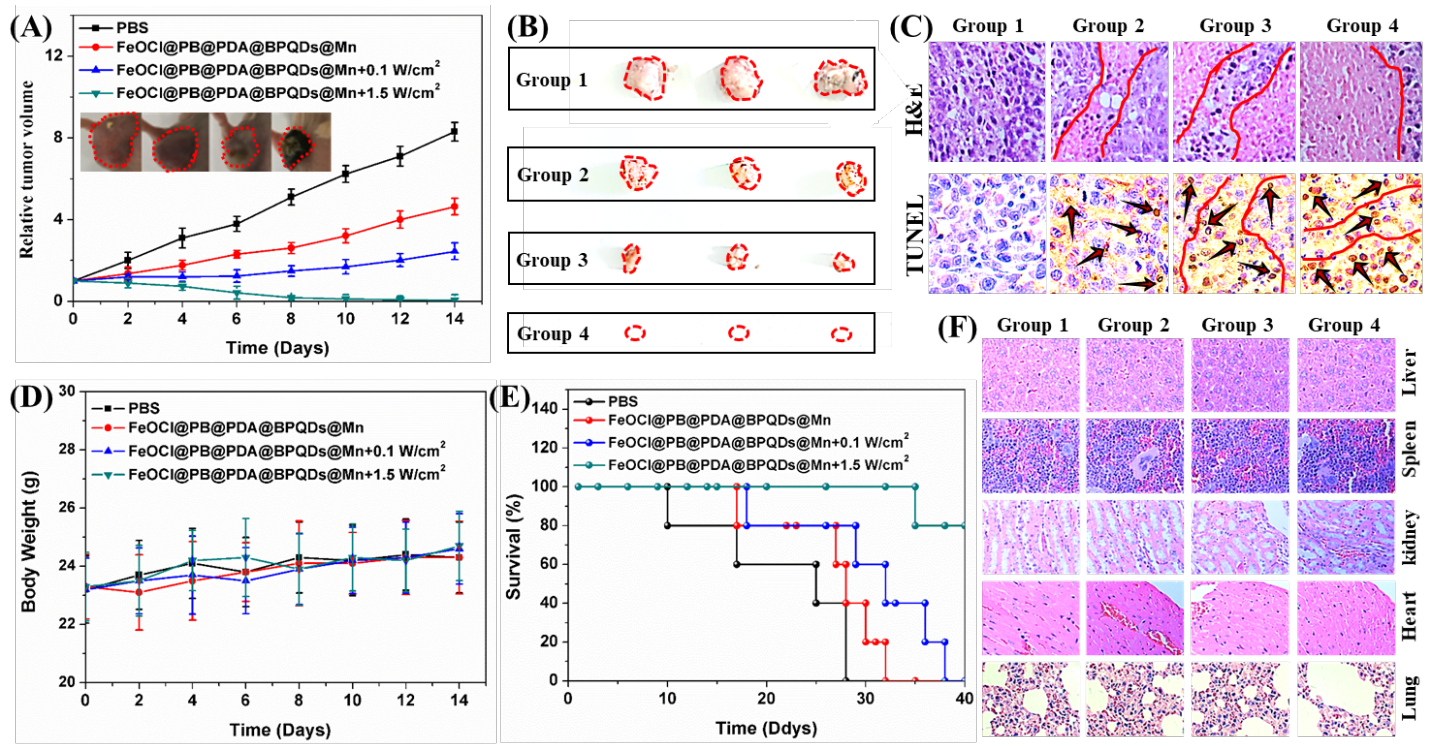

$(\mathbf{G})$

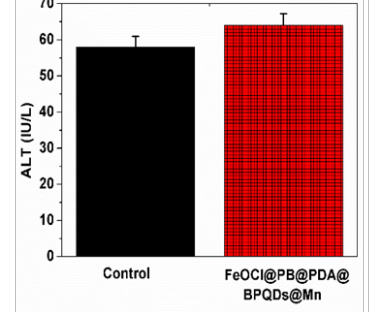

$\left(\mathbf{H}_{30}\right.$

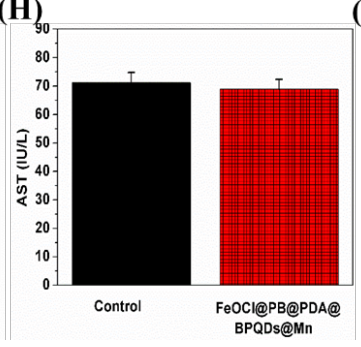

(I)

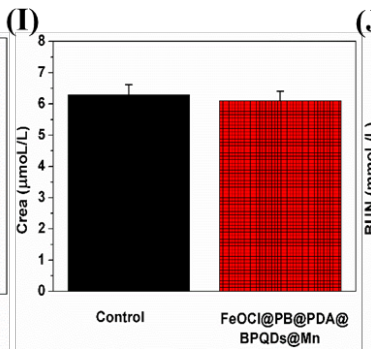

(F) Group 1 Group 2 Group 3 Group 4

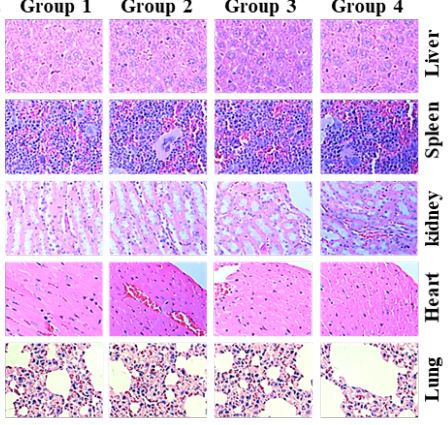

(J)

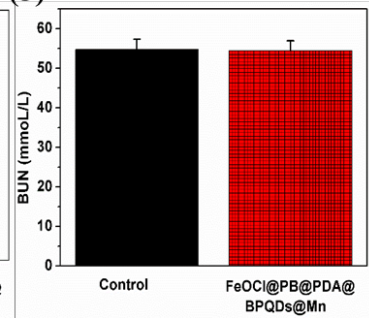

Figure 5. (A) Changes of mice tumor volumes of different treatment groups. Inset: Representative 
photographs of mice tumor at different times after treatment. (B) Photos of the tumors removed from different treatment groups. (C) H\&E and TUNLE staining of tumors collected from different treatment groups. (D) Body weight changes of mice at different times after treatment. (E) Survival rates of mice after various treatments as indicated. (F) H\&E stained images of major organs collected from different treatment groups. (G-J) Serum biochemistry data for mice injected with PBS and FeOCl@PB@PDA@BPQDs@MnNCs at day 14.

For the body weight of the mice in the four groups, no significant changes were detected during the period of treatment (Figures 5D). All mice in the CDT/PDT/PTT group survived for $>35$ days, while the mice in the other three control groups only showed the life spans of about 10-38 days (Figures 5E). Additionally, no significant damage was detected in liver, heart, spleen, kidney, and lung collected from the mice at the 14th day followed various treatments, after being stained by H\&E (Figures 5F). Moreover, the toxicity of FeOCl@PB@PDA@BPQDs@Mn NCs were detected by the analysis of biochemical blood tests, including two important hepatic indicators (alanine aminotransferase (ALT) and aspartate aminotransferase (AST)) and two indicators for kidney functions (creatinine (Crea) and blood urea nitrogen (BUN)) $[49,50]$. These results are shown in Figures 5G-J and reveal that these indicators were similar for the mice exposed to FeOCl@PB@PDA@BPQDs@Mn NCs and for the control mice. Taken together, these in vivo results further demonstrated the biocompatibility of FeOCl@PB@PDA@BPQDs@Mn NCs and their ability to enhance CDT, PDT, and PTT by relieving hypoxia based symptoms in tumor cells.

\section{Conclusions}

In this study, we fabricated multifunctional FeOCl@PB@PDA@BPQDs@Mn NCs as potential therapeutic agents for the first time. The preparation was based on combining the multifunctional properties of new Fenton-like catalyst $/ \mathrm{O}_{2}$-evolving agent $\mathrm{FeOCl}$ together with a photothermal/continuous $\mathrm{O}_{2}$-evolving/MR imaging contrast agent PB, highly biocompatible/photothermal/biodegradable polymer PDA, photosensitizer BPQDs, and continuous $\mathrm{O}_{2}$-evolving/MR imaging contrast agent $\mathrm{Mn}^{2+}$ NCs. The FeOCl@PB@PDA@BPQDs@Mn NCs could effectively catalyze abundant $\mathrm{H}_{2} \mathrm{O}_{2}$ into $\mathrm{O}_{2}$ and $\bullet \mathrm{OH}$ for PDT and CDT in the TME. In addition, due to its 
broad NIR absorption properties, FeOCl@PB@PDA@BPQDs@Mn NCs were capable to serve as a photothermal agents whereby upon irradiation with a $650 \mathrm{~nm}$ laser, they demonstrated a photothermal efficiency conversion rate of up to $28.4 \%$. Moreover, the potential for FeOCl@PB@PDA@BPQDs@Mn NCs as multimodal imaging agents was verified by the strong signals observed for MR, PA, and US at the tumor sites, which was applied to detect the biodistribution of NCs as well as their therapeutic efficacy conveniently. The novel nanotheranostic system provided a promising strategy for TME-responsive treatment with enhanced inhibitory effects on tumors and limited side effects on normal healthy tissues.

\section{Acknowledgement}

This work was supported by the Jiangsu six category outstanding talent (2012-NY-031), Jiangsu province science and technology support plan (BE2015367), and the Villum Fonden, Denmark, Project No. 13153. M.Z. B.S, and T.Z. would like to thank the CSC for its generous support.

\section{Conflicts of interest}

The authors declare no conflict of interest.

\section{References}

[1] X. Zhang, H. Xie, Z. Liu, Angew. Chem., Int. Ed. 54 (2015) 3653-3657.

[2] M. Qiu, D. Wang, et al., Proc. Nat. Acad. Sci. 115 (3) (2018) 501-506.

[3] M. Zhang, W. Wang, et al., Chem. Eur. J. 24 (2018) 12890-12901.

[4] C. Zhu, F. Xu, et al., Chem. Eur. J. 22 (2016) 7357-7362.

[5] Y.J. Yuan, S. Yang, P. Wang, Chem. Commun. 54 (2018) 960-963.

[6] L. Ye, H. Li, Z.F. Chen, J.B. Xu, ACS Photonics 3 (2016) 692-699.

[7] A.Q. Lin, Y.G. Sun, H. Zhang, et al., Appl. Therm. Eng. 142 (2018) 779-792.

[8] W. Chen, J. Ouyang, H. Liu, M. Chen, K. Zeng, Adv. Mater. 29 (2017) 1603864.

[9] M. Zhang, W. Wang, Y. Cui, N.L. Zhou, J. Shen, Int. J. Nanomed. 13 (2018) 2803-2819.

[10] J. Shao, H. Xie, H. Huang, Nat. Commun. 7 (2016) 12967.

[11] H. Wang, X. Yang, W. Shao, J. Am. Chem. Soc. 137 (2015) 11376-11382.

[12] Q. Jia, J. Ge, W. Liu, Adv. Mater. 30 (2018) 1706090.

[13] Z. Tang, H. Zhang, Y. Liu, Adv. Mater. 29 (2017) 1701683. 
[14] D.B. Zorov, M. Juhaszova, S.J. Sollott, Physiol. Rev. 94 (2014) 909-950.

[15] G. Yang, R. Zhang, C. Liang, Small 14 (2018) 1702664.

[16] T. Zheng, W. Wang, et al., Theranostics 9 (17) (2019) 5035-5048.

[17] C. Yue, Y. Yang, et al., Theranostics 6 (13) (2016) 2352-2366.

[18] Z.L. Yang, W. Tian, Q. Wang, Adv. Sci. 5 (2018) 1700847.

[19] M. Yu, X. Xu, Y. Cai, Biomaterials 175 (2018) 61-71.

[20] C.C. Huang, W.T. Chia, M.F. Chung, J. Am. Chem. Soc. 138 (2016) 5222-5225.

[21] C.P. Liu, T.H. Wu, C.Y. Liu, Small 13 (2017) 1700278.

[22] J. Guo, Y. Wang, M. Zhao, Sens. Actuators, B 297 (2019) 126739.

[23] J. Kim, H.R. Cho, H. Jeon, J. Am. Chem. Soc. 139 (2017) 10992-10995.

[24] Y. Cao, X. Meng, D. Wang, ACS Appl. Mater. Interfaces 10 (2018) 17732-17741.

[25] X.Y. Jia, H. Zhang, Q. Zheng, Appl. Therm. Eng. 152 (2019) 79-91.

[26] J. Peng, Q. Yang, W. Li, ACS Appl. Mater. Interfaces 9 (2017) 44410-44422.

[27] Y. Wang, X. Pang, J. Wang, J. Mater. Chem. B 6 (2018) 2460-2473.

[28] W. Chen, K. Zeng, H. Liu, Adv. Funct. Mater. 27 (2017) 1605795.

[29] A.E. ElMetwally, G. Eshaq, F.Z. Yehia, ACS Catal. 8 (2018) 10668-10675.

[30] M. Sun, C. Chu, F. Geng, Environ. Sci. Technol. Lett. 5 (2018) 186-191.

[31] M. Zhang, T. Zheng, et al., Chem. Eur. J. 373 (2019) 1054-1063.

[32] B. Poinard, S.Z.Y. Neo, E.L.L. Yeo, ACS Appl. Mater. Interfaces 10 (2018) 21125-21136.

[33] Z. Dong, H. Gong, M. Gao, D.D. Zhu, X.Q. Sun, Theranostics 6 (2016) 1031-1042.

[34] Q. Jia, J. Ge, W. Liu, X. Zheng, S. Chen, Adv. Mater. 30 (2018) 1706090.

[35] S.H. Kim, I. In, S.Y. Park, Biomacromolecules 18 (2017) 1825-1835.

[36] J. Lu, F. Fu, L. Zhang, Chem. Eng. J. 346 (2018) 590-599.

[37] Y. Liu, K. Ai, L. Lu, Chem Rev. 114 (2014) 5057-5115.

[38] M. Zhang, W. Wang, N.L. Zhou, Carbon 118 (2017) 752-764.

[39] D.K. Roper, W. Ahn, M. Hoepfner, J. Phys. Chem. Cm, 111 (2007) 3636-3641.

[40] S. Zhang, W. Guo, J. Wei, C. Li, X. Liang, ACS Nano 11 (2017) 3797-3805.

[41] C.M. Hessel, V.P. Pattani, et al., Nano Lett. 11 (2011) 2560-2566.

[42] Y. Yang, H. Wu, B. Shi, Part. Part. Syst. Charact. 32 (2015) 668-679.

[43] T. Zheng, T. Zhou, X. Feng, J. Shen, M. Zhang, ACS Appl. Mater. Interfaces 11 (2019) 31615-31626.

[44] G. Yang, P.Yang, et al., Adv. Funct. Mater. 27 (2017) 1700371.

[45] J. Zhang, X.L. Jiao, Y.G. Xia, Chem. Eur. J. 22 (2016) 9321-9329.

[46] H. Shi, R. Yan, L. Wu, Acta Biomater. 72 (2018) 256-265.

[47] Y. Zhao. W. Song, D. Wang, ACS Appl. Mater. Interfaces 7 (2015) 14231-14242.

[48] T. Lin, X. Zhao, et al., Theranostics, 8 (4) (2018) 990-1004.

[49] T. Yu, X. Huang, et al., Appl. Mater. Today 16 (2019) 351-366.

[50] H. Cheng, P. Yuan, et al., Appl. Mater. Today 16 (2019) 120-131. 